\title{
Lifted Evaluation of mp-MIQP Solutions
}

\author{
Alexander Fuchs, Daniel Axehill and Manfred Morari
}

\section{Linköping University Post Print}

\section{Tweet}

N.B.: When citing this work, cite the original article.

Alexander Fuchs, Daniel Axehill and Manfred Morari, Lifted Evaluation of mp-MIQP Solutions, 2015, IEEE Transactions on Automatic Control, (60), 12, 3328-3331. http://dx.doi.org/10.1109/TAC.2015.2417853

(C2015 IEEE. Personal use of this material is permitted. However, permission to reprint/republish this material for advertising or promotional purposes or for creating new collective works for resale or redistribution to servers or lists, or to reuse any copyrighted component of this work in other works must be obtained from the IEEE.

\section{http://ieeexplore.ieee.org/}

Postprint available at: Linköping University Electronic Press

http://urn.kb.se/resolve?urn=urn:nbn:se:liu:diva-124516 


\title{
Lifted evaluation of mp-MIQP solutions
}

\author{
Alexander Fuchs, Daniel Axehill, Manfred Morari
}

\begin{abstract}
This paper presents an efficient approach for the evaluation of multi-parametric mixed integer quadratic programming (mp-MIQP) solutions, occurring for instance in control problems involving discrete time hybrid systems with quadratic cost. Traditionally, the online evaluation requires a sequential comparison of piecewise quadratic value functions. We introduce a lifted parameter space in which the piecewise quadratic value functions become piecewise affine and can be merged to a single value function defined over a single polyhedral partition without any overlaps. This enables efficient point location approaches using a single binary search tree. Numerical experiments with a power electronics application demonstrate an online speedup up to an order of magnitude. We also show how the achievable online evaluation time can be traded off against the offline computational time.
\end{abstract}

Index Terms-Explicit MPC; Control of discrete time hybrid systems; Control of constrained systems

\section{INTRODUCTION}

The main motivation for this work is control of discrete-time hybrid systems [1], [2], [3] using Model Predictive Control (MPC) with quadratic cost [4]. The paper considers the parametric solution to the MPC problem, where the optimal control is computed offline for a set of initial states to reduce the online computational effort [5], [6]. For hybrid systems with quadratic cost, the offline computation requires to solve a multi-parametric Mixed Integer Quadratic Programming (mp-MIQP) problem [3]. Solutions to mp-MIQPs have been proposed based on the solution of Mixed Integer Nonlinear Programming problems [7], on the enumeration of all switching sequences [3], on dynamic programming [8], and on parametric branch and bound [9].

For efficient evaluation, a parametric solution needs to be stored in a suitable data structure. The

A. Fuchs is with the Research Centre for Energy Networks, Swiss Federal Institute of Technology (ETH), 8092 Zurich, Switzerland. E-mail: fuchs@fen.ethz.ch .

D. Axehill is with the Division of Automatic Control, Linköping University, 58183 Linköping, Sweden. E-mail: daniel@isy.liu.se .

M. Morari is with the Automatic Control Laboratory, Swiss Federal Institute of Technology (ETH), 8092 Zurich, Switzerland. E-mail: morari@control.ee.ethz.ch . evaluation approaches in [10], [11], [12] are focused on solutions with non-overlapping polyhedral partitions, which can be computed for mp-LP, mp-QP or mp-MILP problems [13]. This covers the MPC problem classes of linear systems with linear or quadratic cost and hybrid systems with linear cost. Efficient data structures for mp-MIQP problems, arising in control problems of hybrid systems with quadratic cost, is the main topic of this paper and a more or less unexplored field. The reason is that the solution is a pointwise minimizer of intersecting piecewise quadratic functions on overlapping polyhedral partitions. Therefore, the boundary between optimal regions is not only defined by hyperplanes but also, in general, by quadratic surfaces. The approach in [14], [15] can be used with arbitrary functions defined on overlapping polyhedral partitions, but requires an online sequential search to compare all of the potentially many overlapping value functions defined for the given parameter vector.

The main contribution in this paper is to show how mp-MIQP solutions can be lifted and then merged to an equivalent piecewise affine function defined over a single polyhedral partition without overlaps. This has a direct impact on the online evaluation time, which can be significantly reduced using a single search tree eliminating the need for the additional sequential search. The paper also outlines a new partial merging scheme. It enables a trade-off between the online and offline complexity of the evaluation of both mp-MILP and mp-MIQP solutions.

\section{EVALUATION PROBLEM FORMULATION}

This section introduces the definitions used to characterize mp-MIQP solutions and states the corresponding evaluation problem.

Definition 1. A polyhedral collection $\mathbb{P}$ in $\mathbb{R}^{n}$ is a finite collection $\mathbb{P}=\left\{\mathrm{P}_{1}, \mathrm{P}_{2}, \ldots, \mathrm{P}_{N}\right\}$ of $|\mathbb{P}|=N$ polyhedra in $\mathbb{R}^{n}$. The i'th polyhedron is referred to as $\mathbb{P}[i]=\mathrm{P}_{i}$. A polyhedral collection whose 
polyhedra are not overlapping is referred to as polyhedral partition $\mathcal{P}$.

Definition 2. The index set $\mathcal{I}_{\mathbb{P}}(x)$ of a polyhedral collection $\mathbb{P}$ with $N$ elements in $\mathbb{R}^{n}$, and a vector $x \in \mathbb{R}^{n}$ is given by

$$
\mathcal{I}_{\mathbb{P}}(x)=\{i \in\{1,2, \ldots, N\}: x \in \mathbb{P}[i]\} .
$$

Definition 3. A collection of quadratic functions $\mathbb{J}$ in $\mathbb{R}^{n}$ is a finite collection $\mathbb{J}=$ $\left\{J_{1}(\cdot), J_{2}(\cdot), \ldots, J_{N}(\cdot)\right\}$,

$$
J_{i}: \mathbb{R}^{n} \rightarrow \mathbb{R}, J_{i}(x)=x^{T} A_{i} x+B_{i}^{T} x+C_{i},
$$

with $A_{i}=A_{i}^{T} \in \mathbb{R}^{n \times n}, B_{i} \in \mathbb{R}^{n}$ and $C_{i} \in \mathbb{R}$. The $i$ 'th quadratic function of $\mathbb{D}$ is referred to as $\mathbb{J}[i](\cdot)=$ $J_{i}(\cdot)$. If all $A_{i}$ are zero matrices, $\mathbb{J}$ is referred to as $a$ collection of affine functions.

Definition 4. $A$ piecewise quadratic function $J_{\mathbb{P}, \mathbb{J}}(\cdot)$ in $\mathbb{R}^{n}$ over a polyhedral collection $\mathbb{P}$ in $\mathbb{R}^{n}$ with a collection of quadratic functions $\mathbb{J}$ in $\mathbb{R}^{n}$ is a map

$$
J_{\mathbb{P}, \mathbb{J}}: \mathbb{R}^{n} \rightarrow \mathbb{R}, \quad J_{\mathbb{P}, \mathbb{J}}(x)=\min _{i \in \mathcal{I}_{\mathbb{P}}(x)} \mathbb{J}[i](x) .
$$

If $\mathbb{J}$ is a collection of affine functions, $J_{\mathbb{P}, \mathbb{J}}(\cdot)$ is referred to as a piecewise affine function.

The multi-parametric solutions can now be characterized as follows:

Lemma 1. The value function of an $m p-M I Q P$ problem can be represented as a piecewise quadratic function. The value function of an mp-MILP problem can be represented as a piecewise affine function.

Proof. See, for instance, [3]. The polyhedral collection $\mathbb{P}$ in general consists of multiple overlapping polyhedral partitions, each corresponding to a fixed value of the problem's integer variables.

The evaluation problem of mp-MIQP solutions requires the solution to the minimization problem in (3), which is implemented as multiple point location problems combined with pairwise comparisons of quadratic functions:

Definition 5. (PL-MIQP): Given a piecewise quadratic function $J_{\mathbb{P}, \mathbb{J}}(\cdot)$ and a vector $x$ in $\mathbb{R}^{n}$, determine an index $i^{*} \in \mathcal{I}_{\mathbb{P}}(x)$ such that

$$
\forall i \in \mathcal{I}_{\mathbb{P}}(x): \quad \mathbb{J}\left[i^{*}\right](x) \leq \mathbb{J}[i](x)
$$

If $J_{\mathbb{P}, \mathbb{I}}(\cdot)$ is a piecewise affine function, the problem is referred to as a (PL-MILP).
In words, among the polyhedra containing the vector, identify the one with the smallest associated function value. The solution of (PL-MIQP) allows to extract the mp-MIQP optimizer associated with the optimal region index $i^{*}$ of the given parameter vector $x$.

\section{LIFTING MP-MIQP SOLUTIONS}

This section introduces a lifting procedure for piecewise quadratic functions, which is the main contribution of the paper. It shows that mp-MIQP value functions have an equivalent piecewise affine representation, known from the solution of (PLMILP) problems, for which there exist efficient evaluation schemes [13].

For the (PL-MILP) case, the description of the piecewise affine function $J_{\mathbb{P}, \mathbb{J}}(\cdot)$ is merged to a function defined over a single polyhedral partition, enabling the construction of a binary search tree [10] for fast online evaluation. These algorithms can however not be directly applied to solve (PLMIQP), i.e. the case with quadratic terms, since $J_{\mathbb{P}, \mathbb{I}}(\cdot)$ is then a non-convex piecewise quadratic function defined on regions that, in general, are partially defined by quadratic boundary constraints.

It will now be shown how an mp-MIQP solution can be lifted to an equivalent piecewise affine formulation in a higher dimensional parameter space. Furthermore, the lifted formulation has the structure of an mp-MILP solution, making the standard state-of-the-art methods designed for (PL-MILP) problems available to (PL-MIQP) problems. Before the main result of this paper is presented, some notations are introduced.

Definition 6. The lifting transformation $L(\cdot)$ of $\mathbb{R}^{n}$, is defined as

$$
\begin{gathered}
L: \mathbb{R}^{n} \rightarrow \mathbb{R}^{l}, \quad l=\frac{n^{2}+3 n}{2}, \\
L(x)=\left[x_{1}, x_{2}, \ldots, x_{n}, x_{1}^{2}, x_{1} x_{2}, \ldots, x_{1} x_{n},\right. \\
\left.x_{2}^{2}, x_{2} x_{3}, \ldots, x_{2} x_{n}, \ldots, x_{n}^{2}\right]^{T} .
\end{gathered}
$$

Definition 7. Given a polyhedral collection $\mathbb{P}$ in $\mathbb{R}^{n}$, the lifted polyhedral collection $\mathbb{P}_{1}=\mathfrak{L}_{\mathfrak{P}}(\mathbb{P})$ in $\mathbb{R}^{l}$, $l=\left(n^{2}+3 n\right) / 2$, is defined as

$$
\begin{aligned}
& \forall i \in\{1, \ldots,|\mathbb{P}|\}: \\
& \quad \mathbb{P}_{1}[i]=\left\{y \in \mathbb{R}^{l}:\left[H_{i}, \mathbf{0}\right] y \leq K_{i}\right\}
\end{aligned}
$$


where $\left(H_{i}, K_{i}\right)$ are the matrices defining

$$
\mathbb{P}[i]=\left\{x \in \mathbb{R}^{n}: H_{i} x \leq K_{i}\right\}
$$

and $\mathbf{0}$ denotes the zero matrix of appropriate dimensions.

Definition 8. Given a collection of quadratic functions $\mathbb{J}$ in $\mathbb{R}^{n}$, the lifted collection of affine functions $\mathbb{J}_{1}=\mathfrak{L}_{\mathfrak{J}}(\mathbb{J})$ in $\mathbb{R}^{l}, l=\left(n^{2}+3 n\right) / 2$, is defined as

$$
\begin{aligned}
& \forall i \in\{1, \ldots,|\mathbb{J}|\}: \\
& \quad \mathbb{J}_{1}[i]: \mathbb{R}^{l} \rightarrow \mathbb{R}, \mathbb{J}_{1}[i](y)=D_{i}^{T} y+E_{i},
\end{aligned}
$$

where

$$
\begin{aligned}
D_{i}= & {\left[B_{i, 1}, B_{i, 2}, \ldots, B_{i, n}, A_{i, 11}, 2 A_{i, 12}, \ldots, 2 A_{i, 1 n},\right.} \\
& \left.A_{i, 22}, 2 A_{i, 23}, \ldots, 2 A_{i, 2 n}, \ldots, A_{i, n n}\right]^{T} \\
E_{i}= & C_{i}
\end{aligned}
$$

are the rearranged parameters of the quadratic functions $\mathbb{J}[i](x)=x^{T} A_{i} x+B_{i}^{T} x+C_{i}$.

The following results show the equivalence of piecewise quadratic functions and the corresponding lifted piecewise affine functions.

Theorem 1. Given a piecewise quadratic function $J_{\mathbb{P}, \mathbb{J}}(\cdot)$ in $\mathbb{R}^{n}$, a lifting transformation $L(\cdot)$ and the lifted collections $\mathbb{P}_{1}=\mathfrak{L}_{\mathfrak{P}}(\mathbb{P})$ and $\mathbb{J}_{1}=\mathfrak{L}_{\mathfrak{J}}(\mathbb{J})$, the piecewise affine function $J_{\mathbb{P}_{1}, \mathbb{J}_{1}}(\cdot)$ satisfies

$$
\forall x \in \mathbb{R}^{n}: \quad J_{\mathbb{P}, \mathbb{J}}(x)=J_{\mathbb{P}_{1}, \mathbb{J}_{1}}(L(x)) \quad .
$$

Proof.

$$
\begin{aligned}
\forall x \in \mathbb{R}^{n}: J_{\mathbb{P}, \mathbb{J}}(x) & =\min _{i \in \mathcal{I}_{\mathbb{P}}(x)} \mathbb{J}[i](x) \\
& =\min _{i \in \mathcal{I}_{\mathbb{P}_{1}}(L(x))} \mathbb{J}[i](x) \\
& =\min _{i \in \mathcal{I}_{\mathbb{P}_{1}}(L(x))} \mathbb{J}_{1}[i](L(x)) \\
& =J_{\mathbb{P}_{1}, \mathbb{J}_{1}}(L(x))
\end{aligned}
$$

which follows, omitting details, from Definitions 4 -8 .

Since Theorem 1 states that value functions of mp-MIQP problems can be represented as equivalent piecewise affine functions $J_{\mathbb{P}_{1}, \mathbb{J}_{1}}(\cdot)$, algorithms for efficient evaluation of mp-MILP solutions, such as [13], [10], can now be directly applied.

\section{EVALUATION OF MP-MIQP SOLUTIONS}

The steps of the offline and online procedure for the evaluation of mp-MIQP solutions make use of existing algorithms in MATLAB [13]. This section outlines the steps of the offline and the online procedure, including a discussion of their complexity.

\section{A. Offline computations}

The lifting operation is applied to $\left|\mathbb{P}_{\mathrm{r}}\right|$ polyhedra and quadratic functions of $\left(\mathbb{P}_{\mathrm{r}}, \mathbb{J}_{\mathrm{r}}\right)$, as defined in Definitions 7 and 8. The construction of the lifted piecewise affine function $J_{\mathbb{P}_{1}, \mathbb{J}_{1}}(\cdot)$ is computationally inexpensive. Both the polyhedral collection $\mathbb{P}_{1}$ and the collection of affine functions $\mathbb{J}_{1}$ require only a rearrangement of the data representing the original piecewise quadratic function $J_{\mathbb{P}, \mathbb{J}}(\cdot)$.

After the lifting, the region overlaps of the piecewise affine function $J_{\mathbb{P}_{1}, \mathbb{J}_{1}}(\cdot)$ in $\mathbb{R}^{l}$ are removed. The corresponding algorithm MERGE is shown as pseudocode in Algorithm 1, based on the implementation in [13]. It provides a single polyhedral partition $\mathcal{P}$ and a collection of affine functions $\mathbb{J}_{\mathrm{m}}$, defining the equivalent piecewise affine function $J_{\mathcal{P}, \mathbb{I}_{\mathrm{m}}}(\cdot)$ in $\mathbb{R}^{l}$. Algorithm 1 consists of two nested for-loops, beginning in lines 2 and 4 . The first processing step inside the two for-loops is the construction of the polyhedral collection $\mathbb{Q}$, which requires less than $|\mathbb{P}|^{2}$ feasibility checks of polyhedra. The central and most expensive part of Algorithm 1 is the set difference operation REGIONDIFF, called $|\mathbb{P}|$ times in line 10. A pessimistic upper bound on the number of LPs solved by REGIONDIFF is given in [16]. The bound is exponential in the dimension of $\mathbb{Q}$, with the total number of constraints of all polyhedra as base.

The lifting of mp-MIQP solutions to $\mathbb{R}^{l}$, as in Definition 6, practically squares the problem dimension compared to mp-MILP solutions in the original parameter space $\mathbb{R}^{n}$. The exponential bound on the number of LPs solved by REGIONDIFF suggests that merging lifted mp-MIQP solutions is much more expensive than merging mp-MILP solutions with a similar polyhedral structure. However, with lifted quadratic functions, only very few hyperplanes of $\mathbb{Q}$, originating from the $|\mathbb{Q}|$ function differences in line 5 of Algorithm 1, actually spread along the bilinear dimensions. In other words, because of the special structure of the lifting procedure, only a small amount of additional complexity is introduced 


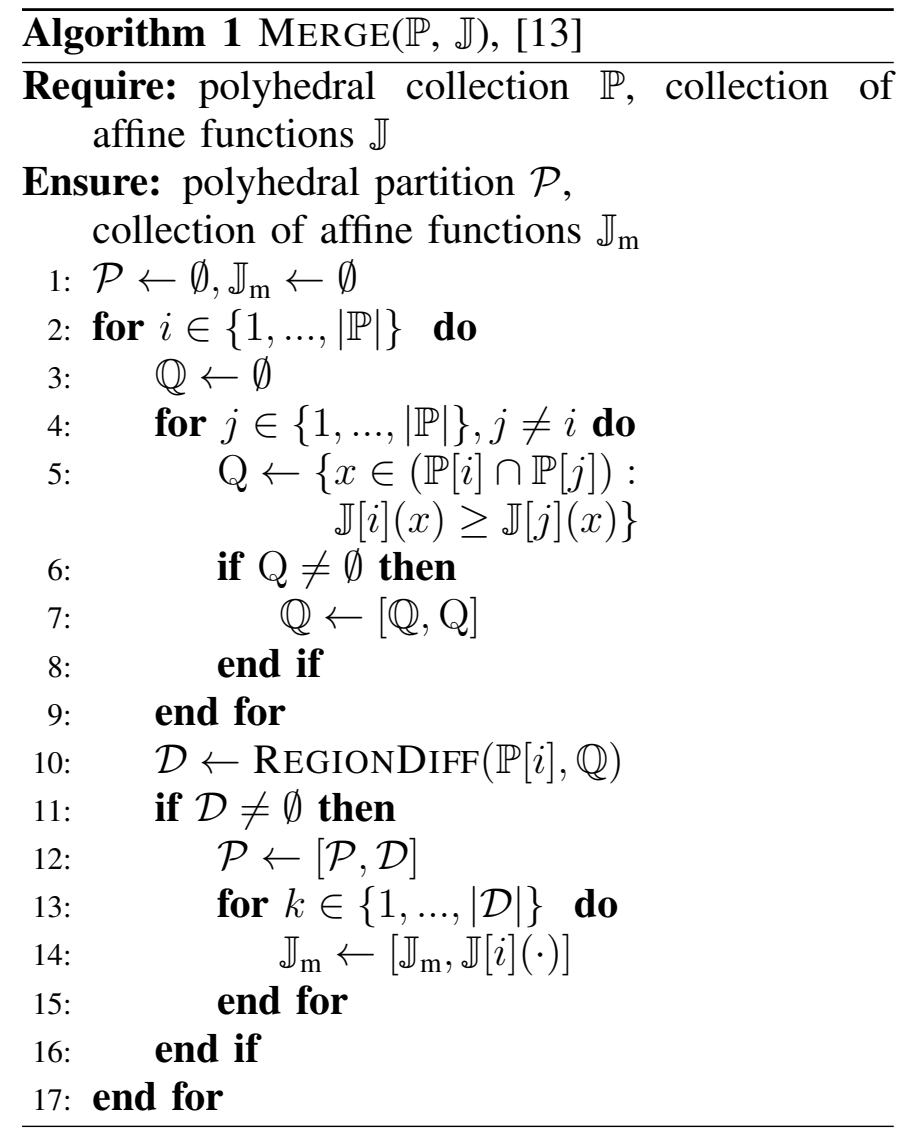

to Merge when applied to a lifted problem (PLMIQP) compared to a problem (PL-MILP) with the same polyhedral collection. Numerical experiments confirm that the total complexity of Algorithm 1 with affine or lifted quadratic functions is similar.

After the merging, the solution of a problem (PLMIQP) reduces to a point location problem in a single polyhedral partition $\mathcal{P}$ and a value function comparison is no longer necessary. An efficient solution to that problem is given by means of a binary search tree. An algorithm to construct a binary search tree using the polyhedra's hyperplanes as decision criteria is given in [10]. A central part of the algorithm is the preprocessing step that determines the relative position of every polyhedron and each of the $n_{\mathrm{h}}$ hyperplanes of the partition, solving up to $2 n_{\mathrm{h}}|\mathcal{P}|$ LPs. Constructing a tree with guaranteed minimum depth might require the solution of an MILP with up to $2|\mathcal{P}|$ binary variables for each node of the tree [17]. The method can also be generalized to trees with more than two children [18], which are particularly suitable for an implementation with multiple processors. Furthermore, the tree can be truncated in order to trade the preprocessing time off against additional online evaluation time [19].
Since neither $n_{\mathrm{h}}$ nor $|\mathcal{P}|$ are directly increased through the additional dimensions from the lifting only little additional complexity is introduced to the tree construction when solving a lifted problem (PL-MIQP) instead of a problem (PL-MILP) with a similar underlying polyhedral collection.

\section{B. Online computations}

After the offline preparation, the solution of (PLMIQP) reduces to the online evaluation of a binary tree. The evaluation is a sequence of vector multiplications [10] that needs to be applied to the lifted vector $y=L(x)$, defined in (5). For mp-MIQP solutions, each region has an associated control law that can now be extracted. A balanced binary tree can execute point location queries in $\log _{2}(|\mathcal{P}|)$ tree node decisions, where $\mathcal{P}$ denotes the merged polyhedral partition [10].

It is of interest how the online evaluation complexity of mp-MIQP solutions compares with and without the preparation through the lifting and merging procedure. While Section VI numerically investigates this question with concrete examples, a basic theoretical comparison is obtained as follows. Consider a piecewise quadratic function $J_{\mathbb{P}, \mathbb{I}}(\cdot)$ in $\mathbb{R}^{n}$ defined over $n_{\text {part }}$ partitions, with the same number of $m$ polyhedra in each partition. Without the lifting and merging, each of the $n_{\text {part }}$ partitions is evaluated with a separate search tree [3]. Then the total number of online operations for the tree evaluations is

$$
N_{\text {ops,no merging }}=n_{\text {part }} \cdot K_{1} \cdot \log _{2}(m),
$$

where $K_{1}$, the number of arithmetic operations per tree node decision, grows linearly with the problem dimension $n$. Additionally, $n_{\text {part }}$ operations are required to find the optimal partition. In comparison, using the lifting and merging procedure, the fully merged partition $\mathcal{P}$ is evaluated with a single tree, requiring

$$
N_{\text {ops,merging }}=K_{2} \cdot \log _{2}(|\mathcal{P}|)
$$

operations. The factor $K_{2}$ is slightly larger than $K_{1}$, depending on the ratio of tree decisions involving the lifted dimensions. It follows that a reduction of the online complexity through the lifting and merging is given whenever

$$
\begin{aligned}
N_{\text {ops,merging }} & <N_{\text {ops,no merging }} \\
\leftrightarrow & |\mathcal{P}|<m^{\frac{K_{1} \cdot n_{\text {part }}}{K_{2}}} \approx m^{n_{\text {part }}} .
\end{aligned}
$$


For mp-MIQP solutions to practical problem instances, one often obtains $|\mathcal{P}| \ll m^{n_{\text {part }}}$, leading to a significant improvement of the evaluation time when using the lifting and merging procedure. This is also confirmed by the examples in Section VI.

\section{TRADING OFFLINE AND ONLINE COMPLEXITY}

This section outlines a practical method for a trade-off between the offline and online complexity for the evaluation of mp-MIQP solutions.

Essentially a trade-off is achieved by merging only subsets of the original mp-MIQP solution, resulting in multiple smaller partitions that need to be evaluated and compared online. Compared to the full merging procedure, this reduces the offline effort and online storage requirements, but also increases the online effort.

One obvious choice for the subsets is to group the overlapping partitions that correspond to different switching sequences of the binary variables into pairs. The pairwise merging of all pairs can be repeated recursively $n_{\mathrm{m}}$ times, until the desired trade-off is achieved. For $n_{\mathrm{m}}=0$, one obtains the traditional evaluation without merging. For $n_{\mathrm{m}}=$ $\left\lceil\log _{2}\left(n_{\text {part }}\right)\right\rceil$, one obtains the evaluation with full merging, as described in Section IV.

\section{NUMERICAL EXPERIMENTS}

In this section, the proposed approach to evaluate mp-MIQP solutions is applied to a practical example of the control of DC-DC converters [20]. All experiments were carried out on a machine with two Intel Xeon E5540 CPUs and 24 GB memory, using MATLAB 2012a and MPT 2.6 [13]. In the example, a mixed logical dynamical system formulation is used and an explicit receding horizon control policy is computed. The equivalent formulation as PWA system has five states, one input, three different dynamics and box constraints on states and inputs. In [20], the system has been controlled using a 1-norm stage cost $\|Q x\|_{1}$. The control approach is applied to the same system formulation, only changing the cost functions to the 2-norm stage cost $x^{T} Q x+u^{T} R u$ with $Q=\operatorname{diag}\left(\left[\begin{array}{lllll}4 & 0.1 & 0 & 0 & 0\end{array}\right]\right)$, $R=0.001$. In the lifted space, which has the dimension $l=20$, it is now possible to merge the overlapping partitions for an efficient implementation of the resulting control policy.
Fig. 1 shows the total number of online operations $N_{\text {ops }}$,

$$
N_{\mathrm{ops}}=(l+1) n_{\mathrm{t}}+\sum_{i=1}^{n_{\mathrm{t}}} N_{\mathrm{ops}, i} .
$$

This includes additions, multiplications and comparisons. The first term in (17) accounts for the value function comparisons in the $l$-dimensional lifted space. The number $N_{\mathrm{ops}, i}$ is the maximum number of operations for a single execution of a tree evaluation. It depends logarithmically on the size of the polyhedral partition and corresponds to the term $K_{1} \cdot \log _{2}(m)$ of the basic complexity estimate in (13). Merging more partitions reduces $n_{\mathrm{t}}$ and increases $N_{\mathrm{ops}, i}$. The motivation of the lifting and merging is that the overall effect is a decrease of the total online complexity $N_{\text {ops }}$, which is confirmed by the numerical experiments below and in [21].

For the shown cases, merging the solution in the lifted space reduces $N_{\text {ops }}$ up to a factor of seven compared to the traditional approach without merging. The factor increases with the prediction horizon $N$ and the number of merging iterations $n_{\mathrm{m}}$. In particular, the evaluation of the completely merged mp-MIQP solution with $N=4$ is faster than the traditional approach to evaluate a solution with $N=2$.

The offline effort for a prediction horizon $N=$ 4 and different number of merging iterations $n_{\mathrm{m}}$ is summarized in Table I. For the preparation of the evaluation using a single search tree, a binary tree was constructed for a partition of 1382120 dimensional polyhedra. The time of the merging operation itself remained relatively small (about 15 minutes), compared to the time of the tree construction (about 36 hours). This also confirms that the lifting does not render the merging problem intractable due to the increased number of dimensions.

\section{CONCLUSION}

The evaluation of mp-MIQP solutions requires a comparison of potentially many overlapping piecewise quadratic value functions defined on polyhedral collections. In this paper it is shown how the quadratic functions and the associated polyhedra can be lifted to a higher dimensional parameter space. It is shown that mp-MIQP solutions in this space have an equivalent representation as a polyhedral piecewise affine function without overlaps. For the 


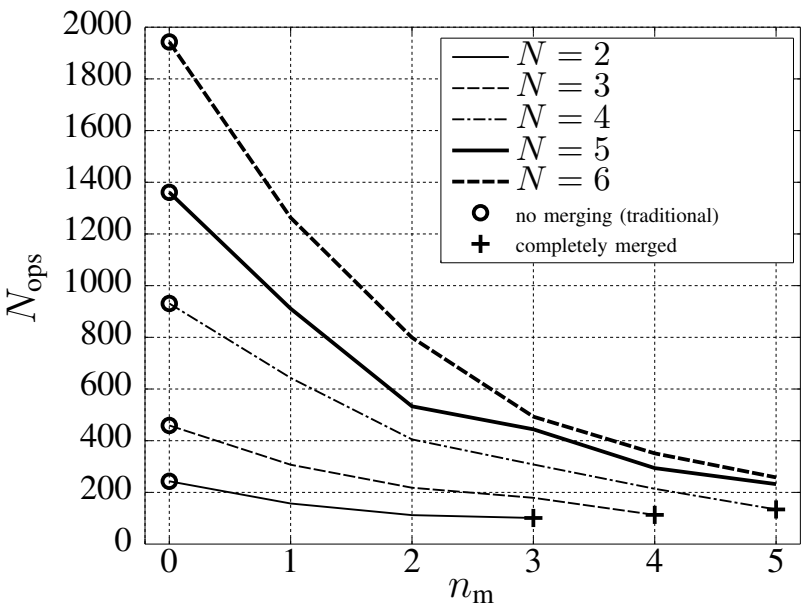

Fig. 1. DC-DC converter problem: Number of online operations $N_{\text {ops }}$ with $n_{\mathrm{m}}$ merging recursions for different prediction horizons $N$.

\begin{tabular}{ccccccc}
$n_{\mathrm{m}}$ & 0 & 1 & 2 & 3 & 4 & 5 \\
\hline \hline$n_{\mathrm{t}}$ & 31 & 16 & 8 & 4 & 2 & 1 \\
$n_{\mathrm{p}}$ & 105 & 104 & 111 & 267 & 1170 & 13821 \\
$n_{\text {store }}$ & 546 & 702 & 954 & 2766 & 12690 & 138402 \\
$t_{\text {merge }}$ & 0 & 1 & 2 & 10 & 51 & 906 \\
$t_{\text {tree }}$ & 8 & 17 & 32 & 99 & 810 & 130000
\end{tabular}

TABLE I

DC-DC CONVERTER PROBLEM WITH PREDICTION HORIZON $N=4$ : NUMBER OF PARTITIONS $n_{\mathrm{t}}$, NUMBER OF POLYHEDRA $n_{\mathrm{p}}$, NUMBER OF FLOATING POINT VALUES STORED IN THE TREE(S) $n_{\text {store }}$, OFFLINE TIME TO MERGE PARTITIONS $t_{\text {merge }}$ [seconds], OFFLINE TIME TO BUILD SEARCH TREES $t_{\text {tree }}$ [seconds], FOR DIFFERENT MERGING RECURSIONS $n_{\mathrm{m}}$.

online evaluation, this enables the use of efficient data structures known from mp-MILP problems, including binary search trees. Furthermore, an algorithm is presented that enables a trade-off between online and offline computational complexity both for mp-MILP and mp-MIQP problems. In the numerical examples, an online speedup up to an order of magnitude is achieved.

\section{REFERENCES}

[1] J. Lunze and F. Lamnabhi-Lagarrigue, Eds., Handbook of Hybrid Systems Control. Cambridge University Press, 2009.

[2] A. Bemporad and M. Morari, "Control of Systems Integrating Logics, Dynamics, and Constraints," Automatica, vol. 35, no. 5, pp. $407-427,1999$.

[3] F. Borrelli, A. Bemporad, and M. Morari, Predictive control for linear and hybrid systems. In preparation, draft at http://www.mpc.berkeley.edu, 2013.

[4] J. Maciejowski, Predictive Control with Constraints. Prentice Hall, June 2001.

[5] B. Bank, J. Guddat, D. Klatte, B. Kummer, and K. Tammer, Non-Linear Parametric Optimization. Berlin: AkademieVerlag, 1982.
[6] E. Pistikopoulos, V. Dua, N. Bozinis, A. Bemporad, and M. Morari, "On-line Optimization via Off-line Parametric Optimization Tools," in International Symposium on Process Systems Engineering, Keystone, USA, Jul. 2000, pp. 183-188.

[7] V. Dua, N. Bozinis, and E. Pistikopoulos, "A Multiparametric Programming Approach for Mixed-Integer Quadratic Engineering Problems," Computers \& Chemical Engineering, vol. 26, pp. 715-733, 2002.

[8] M. Baotić, "Optimal Control of Piecewise Affine Systems A Multi-parametric Approach," Ph.D. dissertation, Automatic Control Laboratory, ETH Zurich, Mar. 2005.

[9] D. Axehill, T. Besselmann, D. Raimondo, and M. Morari, "A parametric branch and bound approach to suboptimal explicit hybrid MPC," Automatica, vol. 50, no. 1, pp. 240-246, Jan. 2014.

[10] P. Tondel, T. Johansen, and A. Bemporad, "Evaluation of piecewise affine control via binary search tree," Automatica, vol. 39, no. 5, pp. 945-950, 2003.

[11] C. Jones, P. Grieder, and S. Rakovic, "A Logarithmic-Time Solution to the Point Location Problem for Parametric Linear Programming," Automatica, vol. 42, no. 12, pp. 2215-2218, Dec. 2006.

[12] Y. Wang, C. Jones, and J. M. Maciejowski, "Efficient point location via subdivision walking with application to explicit MPC," in European Control Conference, Kos, Greece, Jul. 2007.

[13] M. Kvasnica, P. Grieder, M. Baotić, and M. Morari, "MultiParametric Toolbox (MPT)," in Proceedings of the International Workshop on Hybrid Systems: Computation and Control, Philadelphia, USA, Mar. 2004, pp. 448-462.

[14] F. Christophersen, M. Kvasnica, C. Jones, and M. Morari, "Efficient Evaluation of Piecewise Control Laws defined over a Large Number of Polyhedra," in Proc. of the European Control Conference, Kos, Greece, Jul. 2007.

[15] A. Fuchs, D. Axehill, and M. Morari, "On the choice of the Linear Decision Functions for Point Location in Polytopic Data Sets - Application to Explicit MPC," in IEEE Conference on Decision and Control, Atlanta, USA, Dec. 2010, pp. 52835288.

[16] M. Baotić, "Polytopic Computations in Constrained Optimal Control ," Automatika, vol. 50, no. 3, pp. 119 - 134, 2009.

[17] A. Fuchs, C. Jones, and M. Morari, "Optimized Decision Trees for Point Location in Polytopic Data Sets - Application to Explicit MPC," in American Control Conference, Baltimore, USA, Jun. 2010.

[18] M. Mönnigmann and M. Kastsian, "Fast explicit MPC with multiway trees," IFAC World Congress, Sep. 2011.

[19] F. Bayat, T. Johansen, and A. Jalali, "Flexible Piecewise Function Evaluation Methods Based on Truncated Binary Search Trees and Lattice Representation in Explicit MPC," Control Systems Technology, IEEE Transactions on, vol. 20, no. 3, pp. 632-640, May 2012.

[20] T. Geyer, G. Papafotiou, and M. Morari, "Hybrid Model Predictive Control of the Step-Down DC-DC Converter," IEEE Transactions on Control Systems Technology, vol. 16, no. 6, pp. 1112-1124, Nov. 2008.

[21] A. Fuchs, D. Axehill, and M. Morari, "Efficient evaluation of mp-MIQP solutions using lifting," arXiv preprint arXiv:1311.4752, 2013. 